\title{
An Adaptive Noise Reduction Method Based on Improved Dislocation Superposition Method for Abnormal Noise Fault Component of Automotive Engine
}

\author{
Jiaoyi Hou, Pengwei Guo, Aoyu Xu, Dayong Ning $\mathbb{D}^{D}$, Shengtao Chen, Zengmeng Zhang, \\ Yongjun Gong, Yinglong Chen, Hao Tian, and Hongwei Du \\ National Center for International Research of Subsea Engineering Technology and Equipment, Dalian Maritime University, \\ Dalian, China \\ Correspondence should be addressed to Dayong Ning; ningdayongning@163.com
}

Received 30 June 2021; Revised 28 September 2021; Accepted 30 September 2021; Published 21 October 2021

Academic Editor: Thanh-Phong Dao

Copyright (c) 2021 Jiaoyi Hou et al. This is an open access article distributed under the Creative Commons Attribution License, which permits unrestricted use, distribution, and reproduction in any medium, provided the original work is properly cited.

\begin{abstract}
The acoustic signal generated by mechanical motion contains the information of its motion state, but when the signal-to-noise ratio (SNR) is low, the accuracy of real-time monitoring mechanical motion state by the acoustic signal is low. This study proposes an adaptive noise reduction method based on the dislocation superposition method (DSM), which can realize the adaptive noise reduction and the extraction of fault a component from the automobile engine abnormal noise signal of low SNR. Firstly, the wavelet coefficients of engine abnormal noise signal are obtained by continuous wavelet transform (CWT), and the fault feature points of the abnormal noise signal in each period are extracted by setting hard threshold function, window function, and feature points extraction algorithm. Then, the signal segments containing fault components are obtained by using the position of feature points to extend the length of the fault component forward and backward, respectively, and Pearson's correlation is calculated by traversal to determine the starting superposition point of each signal segment containing fault components. Finally, the signal segments of the odd group and even group are selected for superposition calculation. When the superposition stop condition is not satisfied, the number of superpositions increased until the stop condition is satisfied, and the superposition signal can be used as a fault component. The experimental results show that, compared with the improved DSM, this method has a good effect on the noise reduction and extraction of fault components of automobile engine cylinder knocking fault, and the effectiveness of this method is verified. This method is used to reduce the noise and extract the fault components of automobile engine cylinder missing fault and knock fault, and good results are obtained.
\end{abstract}

\section{Introduction}

At present, cars play an important role in human life and are a necessary condition for human travel. The engine is an important part of the automobile, and its structure is complex [1]. In the working process of the engine, due to wear, fatigue, aging, and other factors, the engine failure may cause serious economic losses and even casualties [2, 3]. When the engine failure produces an abnormal noise signal, the higher SNR of the abnormal noise signal will make engine fault diagnosis easier and more accurate, so it is necessary to improve the SNR of the engine abnormal noise signal [4].
The traditional noise reduction methods mainly include Wiener filtering, spectral subtraction, and minimum mean square deviation, but the effectiveness of noise reduction for the nonstationary signal and short-term transient signal is significantly reduced $[5,6]$. The commonly used noise reduction methods mainly include wavelet transform (WT) [7], empirical mode decomposition (EMD) [8], local mean decomposition (LMD) [9], DSM [10], etc.

As a widely used signal processing tool, WT has strong multiresolution analysis ability in the time domain and frequency domain. The wavelet denoising generally uses setting the wavelet coefficient threshold to eliminate the noise information and then uses the inverse WT to 
reconstruct the signal from the threshold wavelet coefficient [11]. Li [12] used the wavelet decomposition and reconstruction algorithm to decompose and reduce noise and reconstruct and analyze the spectrum of bearing vibration signal. Experiment shows that the wavelet noise reduction method is very suitable for fault frequency detection of weak vibration signal of the rolling bearing in low SNR cases. Moshrefi et al. [13] used WT and adaptive filtering to denoise the knock signal of the internal combustion engine. This method is applied to real knock signal, which showed superiority compare to previous works and led to a $13.2 \%$ improvement in detection accuracy. Kai et al. [14] proposed a wavelet denoising method based on improved threshold function by studying soft threshold function and hard threshold function. By this method, the feature of fault signal is highlighted and the fault diagnosis effect was improved. Wen and You [15] used the wavelet decomposition and reconstruction algorithm to denoise the fault signal of highspeed rolling bearing, and the experimental data showed that most of the noise has been filtered out compared with the original data. Although wavelet denoising has good processing ability for nonstationary signals, the selection of wavelet basis function is very difficult. Different wavelet basis functions have different denoising effects. To solve this problem, Huang et al. [16] proposed the EMD, which can efficiently decompose nonlinear and nonstationary signals without any set of basis functions. Sun et al. [17] used the wavelet threshold noise reduction algorithm based on EMD to solve the problem of complex centrifugal pump vibration signals. The experimental results show that the wavelet soft threshold denoising algorithm based on EMD decomposition has a better noise reduction effect when the centrifugal pump vibration signal is used as the noise reduction object. Ren and Liu [18] proposed an adaptive reduction noise and feature extraction algorithm based on improved EMD and verified the effectiveness and feasibility of the method by simulation signals and examples. Although EMD has been successfully applied in the field of signal noise reduction, it also has its limitations, such as the frequent occurrence of modal mixing. In view of the shortcomings of EMD, Wu and Huang [19] proposed ensemble empirical mode decomposition (EEMD) in 2009. EEMD adds finite amplitude Gauss white noise to the signal, and then the signal with the white noise is decomposed as a whole, to effectively suppress the mode mixing of EMD. However, this method can only reduce modal mixing to some extent.

In recent years, scholars have also proposed some other signal noise reduction methods. Gao et al. [20] proposed a new method based on LMD and wavelet denoising to analyze the signals of bearing outer ring, inner ring, and ball. However, LMD has the phenomenon of modal mixing, which reduces the accuracy of signal decomposition and affects the accuracy of the noise reduction signal. Dayong et al. [21] proposed DSM based on random decrement technique in 2015. Compared with other methods, DSM only calculates in time domain. In the superposition process, the method does not destroy the correlation components in the mixed signal and effectively avoids the modal mixing. In 2019, Dayong et al. [10] used improved DSM to automatically extract engine fault components. Although the improved DSM can automatically extract the fault components of the automotive engine quasiperiodic signal, this method needs to use the pulse number of the encoder to determine the starting superposition point of each quasiperiodic fault signal. In practical applications, automobile engines are not suitable for installing encoders, and encoders are prone to failure when subjected to severe impact, so the improved DSM is limited in some practical applications.

To overcome the above problems, this paper proposes an adaptive noise reduction and extraction method of engine abnormal noise signal fault components based on improved DSM. The essence of this method is the superposition calculation in the time domain, which avoids the modal mixing and can better deal with the acoustic signal with low SNR. The method can adaptively select the starting superposition points, superposition length, and superposition number. By changing the superposition number, the noise reduction degree of fault components can be changed. Compared with the improved DSM, it not only reduces the use of encoders and improves the practicability of DSM but also makes it more convenient to extract fault components.

\section{DSM Review}

The mathematical expression of the traditional DSM is as follows:

$$
\widehat{S}(n)=\frac{1}{K+1} \sum_{K=0}^{K} S(n+K L),
$$

where $S(n)$ is the original signal, $\hat{S}(n)$ is the signal processed by DSM (named superposition signal), $K$ is the number of superpositions $(K=0,1,2, \ldots)$, and $L$ is the superposition step length (the period of the signal to be processed). Figure 1 shows the graphical description of DSM processing results.

In Figure 1, $\mathrm{S}$ is the target signal; $\mathrm{N}$ is the interference signal; $\mathrm{SN}$ is a mixed signal of $\mathrm{S}$ and $\mathrm{N} ; \mathrm{SN}_{1}, \mathrm{SN}_{2}$, and $\mathrm{SN}_{3}$ are superposition signals obtained by superposition of 5,15 , and 20 times, respectively, using equation (1); The superposition step length $L$ is the period of signal S. Compared with signal $\mathrm{SN}$, the component proportion of target signal in signals $\mathrm{SN}_{1}, \mathrm{SN}_{2}$, and $\mathrm{SN}_{3}$ increases with the increase of superposition times. On the contrary, the proportion of interference signal decreases with the increase of superposition times [22]. Generally, Pearson's correlation coefficient is used to compare the similarity between the target and the superimposed signals to test the DSM processing effect [10].

\section{Adaptive Noise Reduction and Extraction Method of Engine Abnormal Noise Fault Component}

3.1. Influence Factors of DSM. Due to the system error of the engine, the actual speed of the engine is slightly changed, which causes the acoustic signal of the engine to be a quasiperiodic signal. Figure 2 shows the schematic of the quasi-periodic signal. 

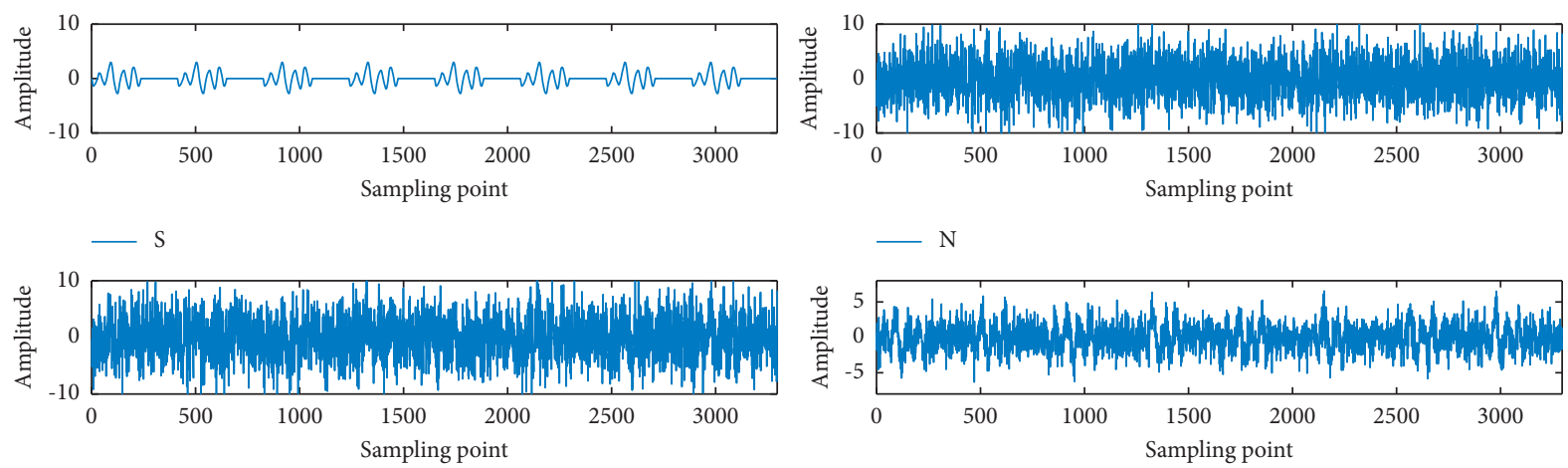

$-\mathrm{SN}$

$-\mathrm{SN}_{1}$
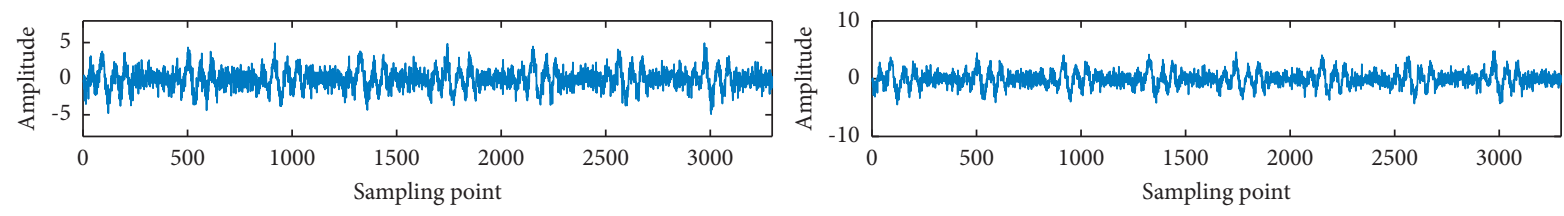

$-\mathrm{SN}_{2}$

$-\mathrm{SN}_{3}$

FIGURE 1: Diagram of DSM. $S$ is the target signal; $N$ is the interference signal; $\mathrm{SN}$ is a mixed signal of $\mathrm{S}$ and $\mathrm{N} ; \mathrm{SN}_{1}, \mathrm{SN}_{2}$, and $\mathrm{SN}_{3}$ are the superposition signals obtained by superposition of 5,15 , and 20 times, respectively, using equation (1). The superposition step length $\mathrm{L}$ is the period of signal $\mathrm{S}$.

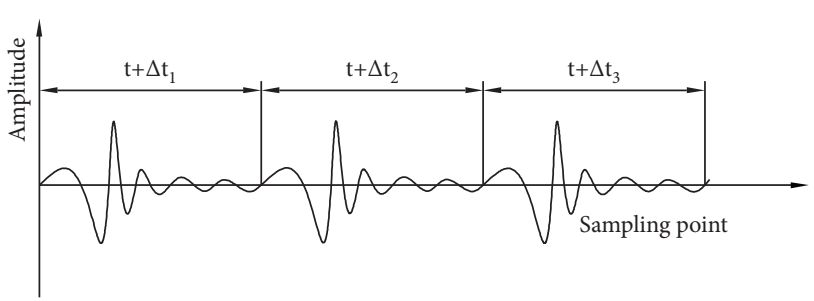

FIGURE 2: Schematic diagram of the quasiperiodic signal. $t$ is the average period and $\Delta t_{1}, \Delta t_{2}$, and $\Delta t_{3}$ are the period deviations, respectively.

When the engine has an abnormal noise impact failure, the fault signal is quasi-periodic, resulting in different superposition step lengths. In the dislocation superposition, it may cause the phase deviation of the impact fault component and even lead to the destruction of the fault component. According to the characteristics of impact failure, it is artificially divided into the "intense change region" and the "stable region," as shown in Figure 3. When the engine impact fault acoustic signal is noise reduction by DSM, due to the less fault energy and information contained in the stable region, to improve the computational efficiency, the intense change region containing more energy and information is separated as the impact fault component $[10,23]$. The noise reduction degree of the fault component also depends on the number of superpositions. Theoretically, the more the number of superpositions is, the better the noise reduction effect is. However, the more the number of superpositions is, the longer the consumed time is. When the number of superpositions reaches a certain amount, the noise reduction effect shows a stable trend. Therefore, the adaptive noise reduction process of engine impact fault acoustic signal is a process of automatically finding the starting superposition points of impact fault acoustic

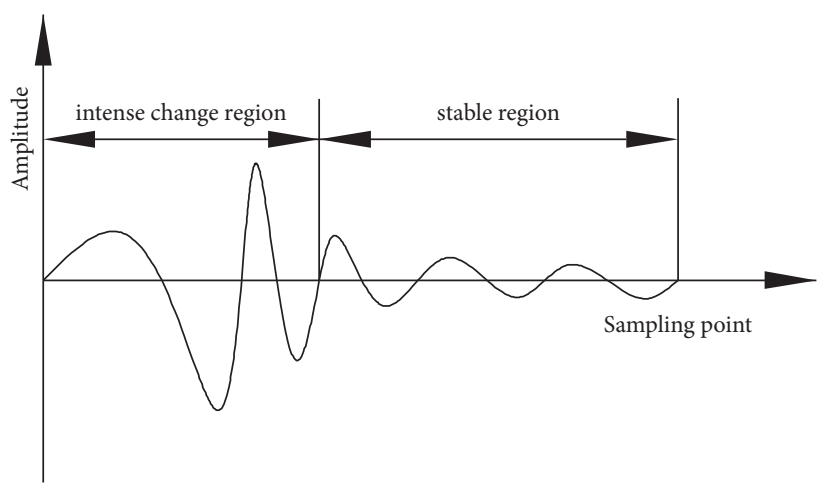

FIgURE 3: The "intense change region" and the "stable region" of impact fault signal.

components, the length of the intense change region, and the number of superpositions.

3.2. Adaptive Noise Reduction and Extraction Method Based on Improved DSM and CWT. Based on CWT and improved DSM, an adaptive noise reduction method for automobile engine fault acoustic signal is proposed. Figure 4 shows the flowchart of the method. The details are as follows.

\subsubsection{Extraction of Feature Point Location of Each Period} Impact Fault Component. The "intense change region" has the characteristics of transient, periodic, and large energy which is not easily submerged by background noise [23]. The mother wavelet which is similar to the waveform of the impact fault component is selected for CWT to obtain wavelet coefficients, and the wavelet coefficients are hard thresholding processes. Finally, the window function and feature points extraction algorithm are used to extract the 


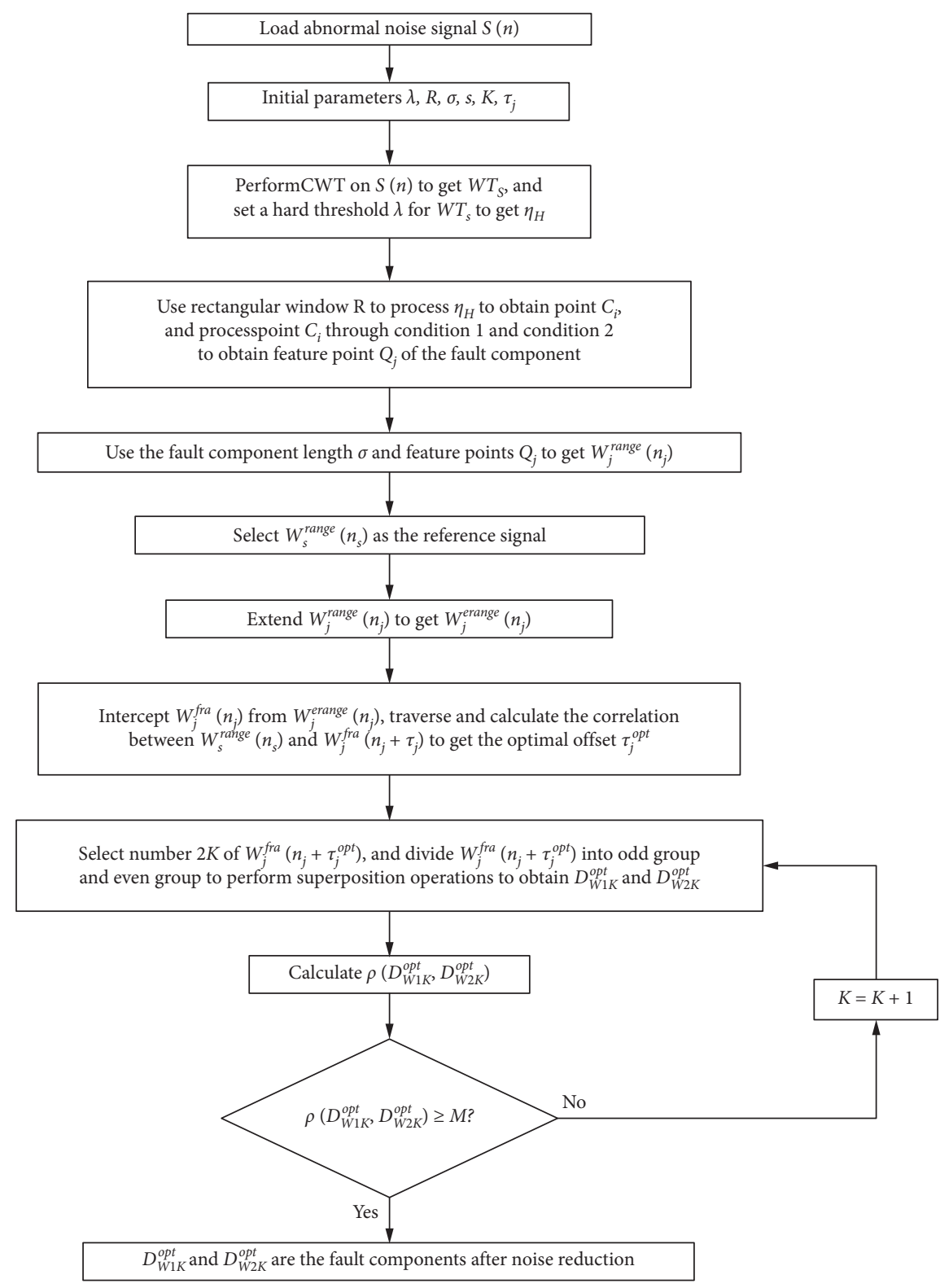

FIGURE 4: The flowchart of the adaptive noise reduction method of automobile engine acoustic signal based on improved DSM and CWT.

feature points location of the impact fault component by using the threshold wavelet coefficient. The specific steps are as follows:

(1) Load the original fault signal $S(n)$, and select the mother wavelet similar to the intense change region of the fault signal to perform CWT on the signal $S(n)$ to obtain the wavelet coefficients $\mathrm{WT}_{S}$.

(2) Eliminate the smaller wavelet coefficients containing interference components in the fault signal through the hard threshold function and retain the larger wavelet coefficients containing the impact fault components. The mathematical model of the hard threshold function is

$$
\eta_{H}\left(\mathrm{WT}_{S}, \lambda\right)= \begin{cases}\mathrm{WT}_{S}, & \left|\left(\mathrm{WT}_{S}\right)\right| \geq \lambda \\ 0, & \left|\left(\mathrm{WT}_{S}\right)\right|<\lambda\end{cases}
$$

where $\eta_{H}\left(\mathrm{WT}_{S}, \lambda\right)$ is the processed wavelet coefficient by hard threshold function, denoted as $\eta_{H} ; \mathrm{WT}_{S}$ are wavelet coefficients; and $\lambda$ is the size of the set hard threshold.

(3) Extract the feature point location of each period fault component.

Two diagonally paired time-frequency points $\left(t_{1}, f_{1}\right),\left(t_{2}\right.$, $f_{2}$ ) are selected to form a rectangular window $R$, and a timefrequency block is selected from the frame of the thresholding wavelet time-frequency diagram by using the rectangular window $R$, which is denoted as $\eta_{H}\left(a_{0}, b_{0}\right)$, where $t_{1}<t_{2}, f_{1}<f_{2}, a_{0}=t_{1}, t_{1}+1 / f_{\mathrm{s}}, \ldots, t_{2}, b_{0}=f_{1}, f_{1}+f_{\mathrm{s}} / 2 a, \ldots$, $f_{2}, f_{s}$ is the sampling frequency, and $a$ is the scale factor of CWT. The maximum point of $\left|\eta_{H}\right|$ in time-frequency block $\eta_{H}\left(a_{0}, b_{0}\right)$ is taken, which is denoted as $C_{0}\left(t_{0}^{\text {spe }}, f_{0}^{\text {spe }}\right)$. Keep the frequency $\left[f_{1}, f_{1}+f_{\mathrm{s}} / 2 a, \ldots, f_{2}\right]$ unchanged, make the 
rectangular window $R$ move one time every $t_{2}$ along the time axis direction, and constantly frame time-frequency block $\eta_{H}\left(a_{i}, b_{i}\right)$ one after another, and the maximum point of $\left|\eta_{H}\right|$ in the time-frequency block $\eta_{H}\left(a_{i}, b_{i}\right)$ is denoted as $C_{i}\left(t_{i}^{\mathrm{spe}}\right.$, $\left.f_{i}^{\mathrm{spe}}\right)$. Ifthe selected signal length is not an integer multiple of the rectangular windowlength, the maximum value of $i$ is

$$
i=\left[\frac{n_{\mathrm{tot}}}{f_{s} \cdot\left(t_{2}-t_{1}\right)}\right],
$$

where [.] is rounding function and $n_{\text {tot }}$ is the total number of sampling points of the signal $S(n)$.

Let the wavelet coefficient corresponding to the point $C_{i}$ $\left(t_{i}^{\mathrm{spe}}, f_{i}^{\mathrm{spe}}\right)$ be $\eta_{H C_{i}}$, and two condition algorithms are set for $\eta_{H C_{i}}$ to extract feature points. Condition 1: if $\eta_{H C_{i}}=0$, $\left|\eta_{H C_{i+1}}\right|>0, \eta_{H C_{i+2}}=0$, then $C_{i+1}\left(t_{i+1}^{\mathrm{spe}}, f_{i+1}^{\mathrm{spe}}\right)$ is called the feature point of the impact fault component. Condition 2: if $\eta_{H C_{i}}=0,\left|\eta_{H C_{i+1}}\right|>0,\left|\eta_{H C_{i+2}}\right|>0, \ldots,\left|\eta_{H C_{i+n}}\right|>0,\left|\eta_{H C_{i+n+1}}\right|=0$. Take the point $C$ corresponding to $\max \left\{\left|\eta_{H C_{i+1}}\right|,\left|\eta_{H C_{i+2}}\right|, \ldots\right.$, $\left.\left|\eta_{H C_{i+1}}\right|\right\}$ as the feature point of the impact fault component. Finally, all the feature points of the impact fault components are assigned to $Q_{j}\left(t_{j j}, f_{j j}\right)$, so the point set of each of the periodic feature points of the impact fault component is $\left[Q_{1}\right.$, $\left.Q_{2}, \ldots, Q_{j}\right]$. Using each periodic feature point of the fault signal to estimate the period of the fault acoustic signal, the period of the fault signal is approximately $T_{1}=t_{22}-t_{11}$, $T_{2}=t_{33}-t_{22}, \ldots, T_{j-1}=t_{j j}-t_{(j j-1)(j j-1)}$.

3.2.2. Extraction of Impact Fault Component by Superimposing Noise Reduction. Since the feature point of the impact fault component is determined by the intense change region of the impact fault signal, the feature point of the impact fault component is located in a certain location of the intense change region of the impact fault signal. According to the feature point $Q_{j}\left(t_{j j}, f_{j j}\right)$ of the impact fault component, the sampling point corresponding to the feature point is

$$
n_{j j}=t_{j j} \cdot f_{s},
$$

where $n_{j j}$ is the number of sampling points corresponding to the fault feature point, $t_{j j}$ is the time of the feature point of the impact fault component, and $f_{s}$ is the sampling frequency.

The range of the impact fault component of each period is as follows: $(\sigma-1)$ sampling points are extended forward and backward from the $n_{j j}$ th sampling point, and the signal segment is denoted as $W_{i}^{\text {range }}\left(n_{j}\right)$, so $W_{i}^{\text {range }}\left(n_{j}\right)$ includes fault components. $n_{j} \in\left[n_{j j}-\sigma+1, n_{j j}+\sigma-1\right] ; \sigma$ is the length of the intense change region. Due to the interference of background noise, the feature point location of per period is different in the intense change region. Therefore, the intense change regions in $W_{i}^{\text {range }}\left(n_{j}\right)$ may have location offset of different degrees in different periods. As shown in Figure 5, there is offset $\tau$ of different degrees in the intense change

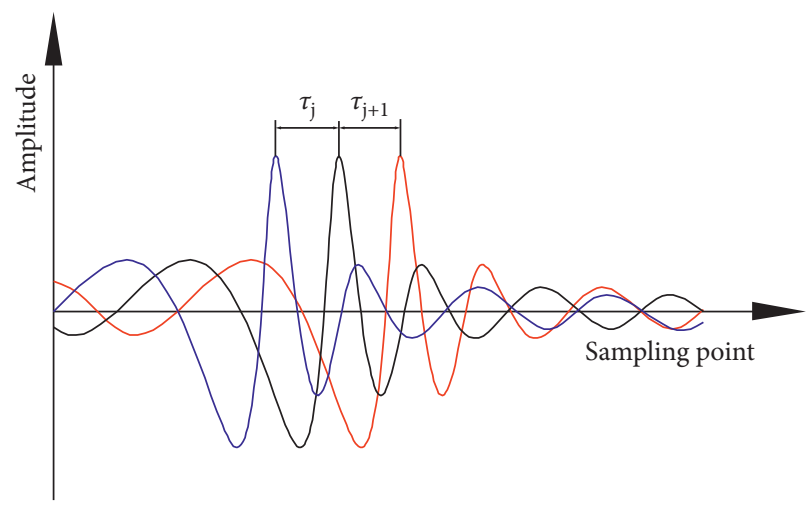

FIgURE 5: The offset $\tau$ of the intense change region in different periods $W_{i}^{\text {range }}\left(n_{j}\right)$.

regions of different periodic $W_{i}^{\text {range }}\left(n_{j}\right)$, which will affect the dislocation superposition effect and lead to inaccurate superposition results.

To solve the problem of the offset of the intense change region, the specific method is as follows.

A segment of $W_{i}^{\text {range }}\left(n_{j}\right)$ containing the fault component is taken as the reference signal, denoted as $W_{s}^{\text {range }}\left(n_{s}\right)$. $W_{i}^{\text {range }}\left(n_{j}\right)$ is extended forward and backward by $b$ sampling points, respectively, to obtain the extended signal segment $W_{i}^{\text {erange }}\left(n_{j}\right), n_{j} \in\left[n_{j j}-\sigma+1-b, n_{j j}+\sigma-1+b\right]$. The continuous intercept length of $(2 \sigma-1)$ sampling points from the first sampling point in $W_{i}^{\text {erange }}\left(n_{j}\right)$ is denoted as $W_{i}^{\text {fra }}\left(n_{j}\right)$. Set $\tau_{j}$ to the offset of $W_{i}^{\text {fra }}\left(n_{j}\right)$ on $W_{i}^{\text {erange }}\left(n_{j}\right)$, and record the offset signal of $W_{i}^{\text {fra }}\left(n_{j}\right)$ as $W_{i}^{\text {fra }}\left(n_{j}+\tau_{j}\right)$, as shown in Figure 6. The subset of $\tau_{j}$ is denoted as $L$, and $L$ is set to $\{0: \sigma-1+b\}$. Find the optimal offset $\tau_{j}^{\text {opt }}$ of the offset $\tau_{j}$ in $W_{j}^{\text {fra }}\left(n_{j}+\tau_{j}\right)$; that is, also find the optimal starting superposition point, where the superposition length is $(2 \sigma-1)$ sampling points.

Traverse all the $\tau_{j}$ values in $L$, and calculate Pearson's correlation coefficient $\rho_{s j}\left(W_{s}^{\text {range }}\left(n_{s}\right), W_{i}^{\text {fra }}\left(n_{j}+\tau_{j}\right)\right)$ of the reference signal segments $W_{s}^{\text {range }}\left(n_{s}\right)$ and $W_{i}^{\text {fra }}\left(n_{j}+\tau_{j}\right)$, respectively; the offset corresponding to the maximum value of $\rho_{s j}\left(W_{s}^{\text {range }}\left(n_{s}\right), W_{i}^{\text {fra }}\left(n_{j}+\tau_{j}\right)\right)$ is the optimal offset $\tau_{i}^{\text {opt }}$. The equation of the proposed method is as follows:

$$
\left(\tau_{1}^{\mathrm{opt}}, \tau_{2}^{\mathrm{opt}}, \ldots, \tau_{j}^{\mathrm{opt}}\right)=\underset{\tau_{j} \in L, j=1,2,3, \ldots}{\arg \max }\left[\rho_{s j}\left(W_{s}^{\text {range }}\left(n_{s}\right), W_{j}^{\text {erange }}\left(n_{j}+\tau_{j}\right)\right)\right],
$$

where $\tau_{j}^{\text {opt }}$ is the best offset; argmax [.] is arguments of the maxima; $W_{s}^{\text {range }}\left(n_{s}\right)$ is the reference signal segment; $W_{i}^{\text {fra }}\left(n_{j}+\tau_{j}\right)$ is the offset signal segment; and $\rho_{s j}\left(W_{s}^{\text {range }}\left(n_{s}\right)\right.$, $\left.W_{i}^{\text {fra }}\left(n_{j}+\tau_{j}\right)\right)$ is to calculate the correlation between $W_{s}^{\text {range }}\left(n_{s}\right)$ and $W_{i}^{\text {fra }}\left(n_{j}+\tau_{j}\right)$.

$2 K$ consecutive signals $W_{i}^{\mathrm{fra}}\left(n_{j}+\tau_{i}^{\mathrm{opt}}\right)$ are selected, which are divided into two groups according to the parity of the sequence number $j$, and each group is superimposed with different times according to the sequence number from low to high. The equation of the proposed method is as follows: 


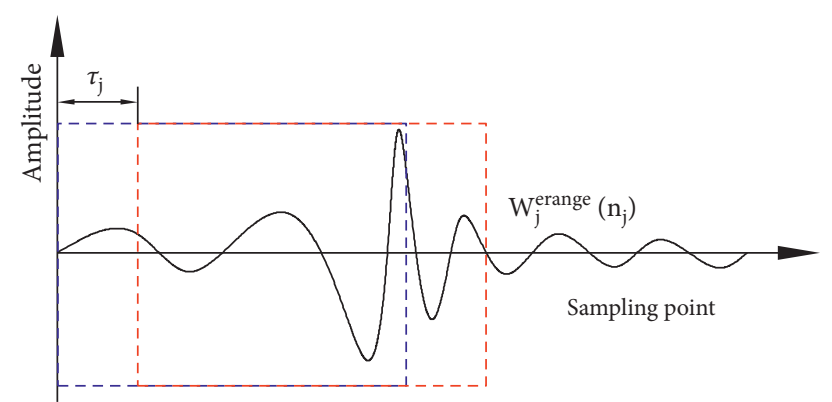

FIgURE 6: $W_{i}^{\text {fra }}\left(n_{j}+\tau_{j}\right)$ signal segment schematic diagram. The part selected by the blue frame is $W_{i}^{\text {fra }}\left(n_{j}\right)$, and the part selected by the red frame is $W_{i}^{\mathrm{fra}}\left(n_{j}+\tau_{j}\right)$.

$$
\left\{\begin{array}{l}
D_{W 1 K}^{\mathrm{opt}}=\frac{1}{K+1}\left[W_{1}^{\mathrm{fra}}\left(n_{1}+\tau_{1}^{\mathrm{opt}}\right)+W_{3}^{\mathrm{fra}}\left(n_{3}+\tau_{j+3}^{\mathrm{opt}}\right)+\cdots+W_{2 k-1}^{\mathrm{fra}}\left(n_{2 k-1}+\tau_{2 k-1}^{\mathrm{opt}}\right)\right], \\
D_{W 2 K}^{\mathrm{opt}}=\frac{1}{K+1}\left[W_{2}^{\mathrm{fra}}\left(n_{2}+\tau_{2}^{\mathrm{opt}}\right)+W_{4}^{\mathrm{fra}}\left(n_{4}+\tau_{4}^{\mathrm{opt}}\right)+\cdots+W_{2 k}^{\mathrm{fra}}\left(n_{2 k}+\tau_{2 k}^{\mathrm{opt}}\right)\right], \\
\rho^{\mathrm{opt}}(K)=\rho\left(D_{W 1 K}^{\mathrm{opt}}, D_{W 2 K}^{\mathrm{opt}}\right),
\end{array}\right.
$$

where $D_{W 1 K}^{\text {opt }}$ is the signal after the superposition of the odd group; $D_{W 2 K}^{\mathrm{opt}}$ is the signal after the superposition of the even group; $K$ is the number of superpositions; $W_{2 K-1}^{\mathrm{fra}}$ $\left(n_{2 K-1}+\tau_{2 K-1}^{\text {opt }}\right)$ is the signal segment of the odd group with fault components; $W_{2 K}^{\text {fra }}\left(n_{2 K}+\tau_{2 K}^{\mathrm{opt}}\right)$ is the signal segment of the even group containing the fault component; $\rho^{\text {opt }}(K)$ is the correlation between the signal after the superposition of the odd group and the signal after the superposition of the even group.

Set the threshold $M$, when $\rho^{\text {opt }}(K) \geq M$, stop the iteration and output the superposition results.

\subsubsection{Parameters Setting}

(1) Hard Threshold $\lambda$ Setting. The hard threshold $\lambda$ is mainly to remove the smaller wavelet coefficients that only contain background noise and retains the larger wavelet coefficients that contain fault components. When the value of $\lambda$ is too small, the smaller wavelet coefficients generated by background noise may be incompletely removed, resulting in unobvious fault periodicity. When the value of $\lambda$ is appropriate, the smaller wavelet coefficients are completely removed, only the larger wavelet coefficients containing fault components are retained, and the fault periodicity is obvious. When the value of $\lambda$ is too large, not only the smaller wavelet coefficients are completely removed, but also part of the larger wavelet coefficients containing fault components will be removed, resulting in a jump in the period of the fault signal. However, the location of the fault component can be found without affecting the superimposing effect. Therefore, the hard threshold $\lambda$ should be as large as possible.

(2) Rectangular Window $R$ Setting. The rectangular window $R$ is composed of two diagonally paired time-frequency points $\left(t_{1}, f_{1}\right),\left(t_{2}, f_{2}\right)$; then, the length of the rectangular window $R$ is $l, l=t_{2}-t_{1}$ and the width $w=f_{2}-f_{1}$. Considering the frequency distribution of the impact fault signal and the length $\sigma$ of the impact fault component, the time of the intense change region is

$$
t_{\sigma}=\frac{\sigma}{f_{s}}
$$

The rectangular window starts from $t=0 \mathrm{~s}$, so $t_{1}=0 \mathrm{~s}$. According to the characteristic frequency of the impact fault signal, which is generally distributed in the middle and low frequency, $f_{1}$ can be set between $0 \mathrm{~Hz}$ and $500 \mathrm{~Hz}$, and $f_{2} \leq 5000 \mathrm{~Hz}$. If $t_{2}$ is too large, it will cause the rectangular window to select multiple fault signal periods, which is not conducive to extracting the fault signal period. If $t_{2}$ is too small, it will increase the amount of calculation. Therefore, the general value range of $l$ is $2 t_{\sigma} \leq l \leq 4 t_{\sigma}$, which corresponds to $2 \sigma$ to $4 \sigma$ sampling points.

(3) The Length of the Intense Change Region $\sigma$. The length $\sigma$ of the intense change region is generally the length of the highamplitude zone of artificially selected fault component. Literature [10] puts forward the general empirical formula of $\sigma$ as

$$
\sigma=\frac{f_{s}}{44100} \times 300
$$

where $f_{s}$ is the sampling frequency of the acoustic signal.

(4) Parameter $b$ Setting. According to the offset of the feature point in the fault component, $b=\sigma-1$ sampling points are selected to minimize the calculation amount finding the optimal offset $\tau_{j}^{\text {opt }}$ more accurately and finding the optimal starting point.

(5) Threshold M Setting. With the increase of superposition number $K$, the correlation coefficient $\rho$ will not always 
increase. When $\rho$ generally increases to 0.9 , it will tend to be stable, and there is an unobvious increase trend [10]. To achieve a better noise reduction effect for the fault component, $M$ should be set to a value between 0.75 and 0.85 , where $M=0.80$.

\section{Experiment Condition}

Figure 7 is the physical diagram of the automobile engine fault detection test bench, which consists of an automobile engine, sound sensor, data acquisition card, encoder, and computer. The engine model is EA211 with the detailed parameters shown in Table 1 . The sound sensor with the frequency range of $20 \mathrm{~Hz}$ to $20 \mathrm{kHz}$ is placed above the cylinder to receive the abnormal sound signal generated by the engine. The data acquisition card uses the USB-6341 produced by National Instruments Company. When collecting the sound signal, the sampling frequency is set to $44100 \mathrm{~Hz}$. The encoder model is ZSP3806GC, and the resolution is $100 \mathrm{P} / \mathrm{R}$. The encoder can synchronously revolve with the crankshaft by fixing it on the front end of the crankshaft through a coupling.

\section{Collection and Processing of Experimental Data}

5.1. Fault Component Extraction of Engine Knocking Cylinder. Figure 8(a) shows the knocking cylinder abnormal noise signal and encoder pulse signal collected under the condition of engine running at $1800 \mathrm{r} / \mathrm{min}$, Figure 8(b) uses $\mathrm{db} 5$ wavelet as the mother wavelet to perform CWT on the knocking cylinder abnormal noise signal to obtain the wavelet time-frequency diagram. The two diagonally paired time-frequency points of the rectangular window $R$ are $(0$, $0),(0.0227,4000)$, respectively. The hard threshold of the wavelet coefficient is $\lambda=0.94$, and the length of the intense change region is calculated by equation (8) to obtain $\sigma=300$, $b=299$. The subset $L$ of offset $\tau_{j}$ is set to $\{0: 598\}$.

Table 2 is the sampling point corresponding to the fault feature point $n_{j j}$ of each periodic obtained by data processing using the above method. Table 3 shows the number of sampling points for each period of impact fault signal calculated by using the feature points of each periodic failure component. It can be seen from Table 3 that the number of sampling points in the $6^{\text {th }}-8^{\text {th }}$ periods is 8708 , which is quite different from the number of sampling points in other periods. This is due to the excessive setting of the hard threshold $\lambda$, which leads to the zeroing of the wavelet coefficients corresponding to the feature points of the fault signal components in the $7^{\text {th }}$ and $8^{\text {th }}$ periods after the hard threshold processing, so the number of sampling points in the $6^{\text {th }}, 7^{\text {th }}$, and $8^{\text {th }}$ periods cannot be obtained, but it does not affect the superposition effect.

Selecting $s=1,299$ sampling points are extended forward and backward from the first feature point 3530 of the fault component to obtain the reference signal segment $W_{1}^{\text {range }}$ $\left(n_{1}\right)$, as shown in Figure 9. Table 4 shows the optimal offset $\tau_{j}^{\mathrm{opt}}$ of each $W_{j}^{\mathrm{fra}}\left(n_{j}+\tau_{j}\right)$ by equation (5), and $W_{j}^{\mathrm{fra}}\left(n_{j}+\tau_{j}^{\mathrm{opt}}\right)$ is obtained according to $\tau_{j}^{\mathrm{opt}}$. The continuous $2 K$ signal segments $W_{j}^{\text {fra }}\left(n_{j}+\tau_{j}^{\text {opt }}\right)$ including fault components are selected and divided into two groups according to the parity of the sequence number $j$ for superposition operation, and the correlation coefficient $\rho^{\text {opt }}(K)$ of the parity group is calculated. Figure 10 shows the variation curve of $\rho^{\text {opt }}(K)$ and the number of superpositions.

When $K=14, \rho^{\text {opt }}(14)=0.8030$, which is greater than the threshold $M$, stopping superimposing. Figure 11 shows the fault component signals $D_{W 114}^{\text {opt }}$ and $D_{W 214}^{\text {opt }}$ after noise reduction.

Figure 12 shows the time-domain diagram of the odd group and even group superimposed signals obtained by superimposing 10 times using the improved DSM. The optimal starting point of superposition is the sampling point of the acoustic signal corresponding to the $28^{\text {th }}$ pulse of each cycle encoder signal. The superposition length is set to 600 sampling points, the odd group and even group are superimposed 10 times to obtain $D_{W 110}^{\text {opt }}$ and $D_{W 210}^{\text {opt }}$, and the correlation reaches 0.8020 .

According to Figures 11 and 12, the length $\sigma=150$ of fault component is accurately extracted. Figure 13 shows the fault components $W_{1}, W_{2}, W_{3}$, and $W_{4}$ extracted from $D_{W 114}^{\mathrm{opt}}, D_{W 214}^{\mathrm{opt}}, D_{W 110}^{\mathrm{opt}}$, and $D_{W 210}^{\mathrm{opt}}$, respectively. The correlation between $W_{1}$ and $W_{3}$ is 0.9029 , and the correlation between $W_{2}$ and $W_{4}$ is 0.9290 , which further verifies that the fault component noise reduction method achieves the same effect compared with the improved DSM.

5.2. Fault Component Extraction of Engine Lacking Cylinder. Figure 14 shows the physical picture of the first cylinder lacking of the automobile engine by pulling out the cylinder line of the first cylinder. Figure 15 shows that the engine produces abnormal noise signal for the first cylinder lacking. The $\mathrm{db} 5$ wavelet is selected as the mother wavelet to perform CWT on the abnormal noise signal of the lack of cylinder. The time-frequency points of the two diagonally paired of the rectangular window $R$ are set as $(0,500),(0.0227,4000)$, respectively. The hard threshold of the wavelet coefficient $\lambda=1, \sigma=300, b=299$, and the subset $L$ of the offset $\tau_{j}$ is set as $\{0: 598\}$.

1415.

Table 5 shows the sampling points corresponding to the feature points of each period of fault component obtained by the above method. Selecting $s=1$, the reference signal $W_{1}^{\text {range }}\left(n_{1}\right)$ is obtained by extending 299 sampling points forward and backward from the $1794^{\text {th }}$ sampling point, as shown in Figure 16. Table 6 shows the optimal offset $\tau_{j}^{\text {opt }}$ of each $W_{j}^{\text {fra }}\left(n_{j}+\tau_{j}\right)$ signal by equation (5), and $W_{j}^{\mathrm{fra}}\left(n_{j}+\tau_{j}^{\mathrm{opt}}\right)$ is obtained according to $\tau_{j}^{\mathrm{opt}}$. Figure 17 shows the variation curve of the correlation coefficient $\rho^{\text {opt }}(K)$ of the superposition signals of the odd group and even group with the number of superpositions $K$. When $K=6, \rho^{\text {opt }}(6)=0.8048$, which is greater than the threshold $M$ and stopping superimposition, $D_{W 16}^{\text {opt }}$ and $D_{W 26}^{\text {opt }}$ of the fault component after noise reduction are got, as shown in Figure 18. 


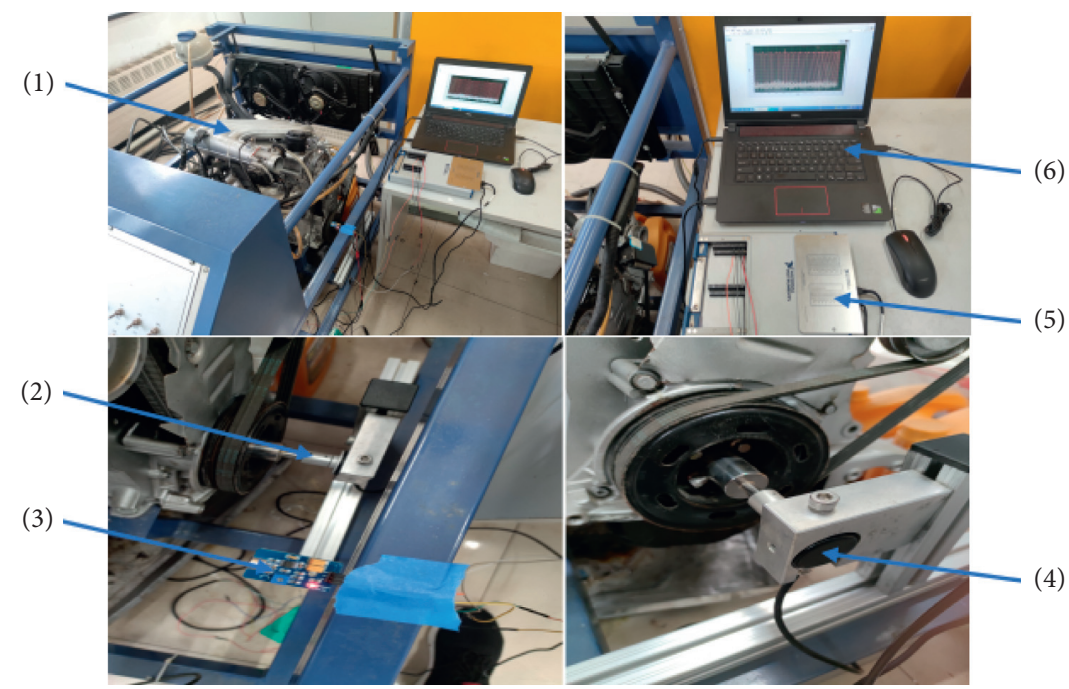

Figure 7: Automobile engine fault detection test bench. (1) Engine; (2) coupling; (3) sound sensor; (4) encoder; (5) data acquisition card; (6) computer.

TABLE 1: Engine parameters.

\begin{tabular}{lc}
\hline Engine type & EA211 \\
Cylinder & 4 \\
Displacement & $1.6 \mathrm{~L}$ \\
Maximum power & $66 \mathrm{~kW}$ \\
Maximum power revolution & $5500 \mathrm{rpm}$ \\
Maximum torque & $132 \mathrm{Nm}$ \\
Maximum torque speed & $3800 \mathrm{rpm}$ \\
\hline
\end{tabular}

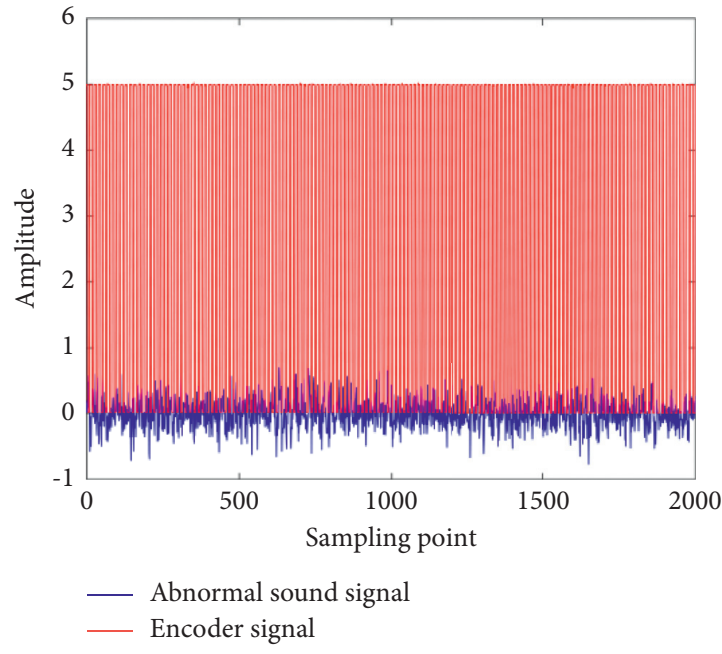

(a)

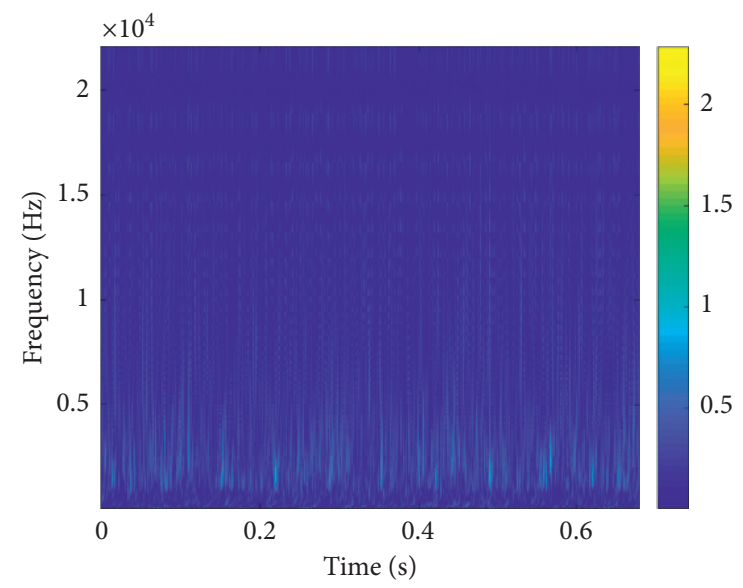

(b)

FIGURE 8: (a) Knocking cylinder and encoder signal collected at $1800 \mathrm{r} / \mathrm{min}$. (b) Wavelet time-frequency diagram.

TABLe 2: The sampling point corresponding to the fault feature points of each period.

\begin{tabular}{lcccccccccccc}
\hline$j$ & 1 & 2 & 3 & 4 & 5 & 6 & 7 & 8 & 9 & 10 & 11 & 12 \\
Sampling point $\left(n_{j j}\right)$ & 3530 & 6734 & 9623 & 12681 & 15538 & 18699 & 27407 & 30568 & 33465 & 36673 & 39590 & 42700 \\
\hline
\end{tabular}


TABLE 3: Error of cylinder knock fault signal period.

\begin{tabular}{|c|c|c|c|c|c|c|c|c|c|c|}
\hline j & 1 & 2 & 3 & 4 & 5 & $6-8$ & 9 & 10 & 11 & 12 \\
\hline Number of sampling points of each period $\left(n_{c}\right)$ & 3204 & 2889 & 3058 & 2857 & 3161 & 8708 & 3161 & 2897 & 3208 & 2917 \\
\hline Actual number of sampling points of each period $\left(n_{a}\right)$ & 3153 & 2953 & 3025 & 2846 & 3174 & 8680 & 3166 & 2917 & 3190 & 2944 \\
\hline Error $(\Delta)$ & 50 & -64 & 33 & 11 & -13 & 28 & -5 & -20 & 18 & -27 \\
\hline
\end{tabular}

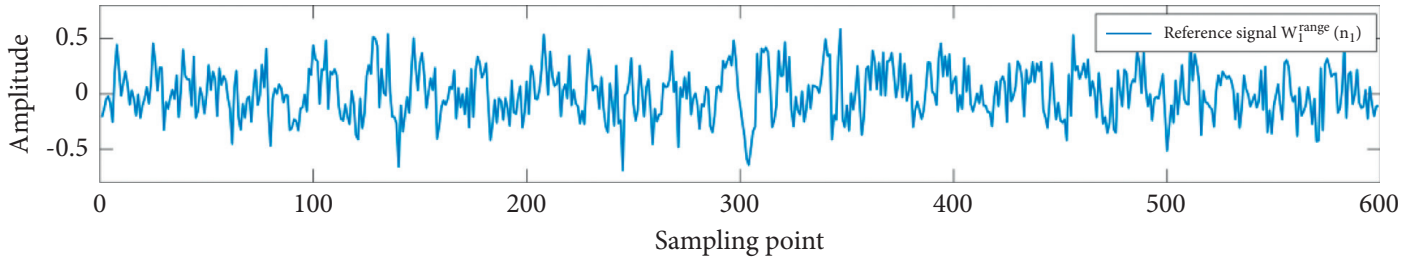

FIGURE 9: Reference signal segment $W_{1}^{\text {range }}\left(n_{1}\right)$.

TABLE 4: The optimal offset $\tau_{j}^{\mathrm{opt}}$ of $W_{j}^{\mathrm{fra}}\left(n_{j}+\tau_{j}\right)$.

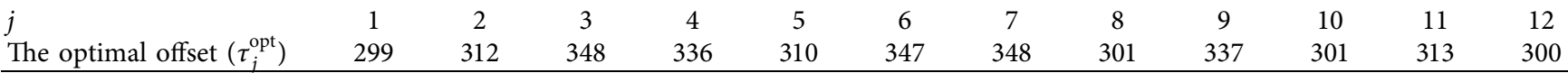

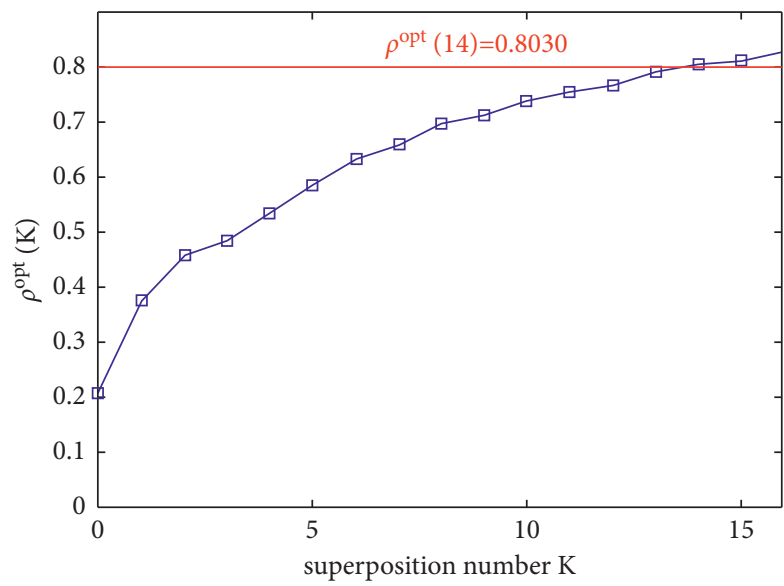

FIGURE 10: The variation curve of $\rho^{\text {opt }}(K)$ with the increase of the superimposition number $(K)$.

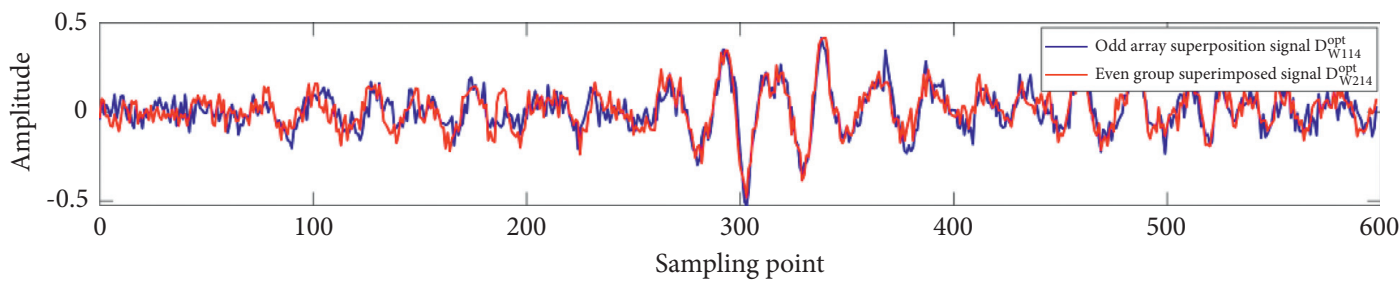

FIGURE 11: Odd and even groups superposition signals after 14-time superposition.

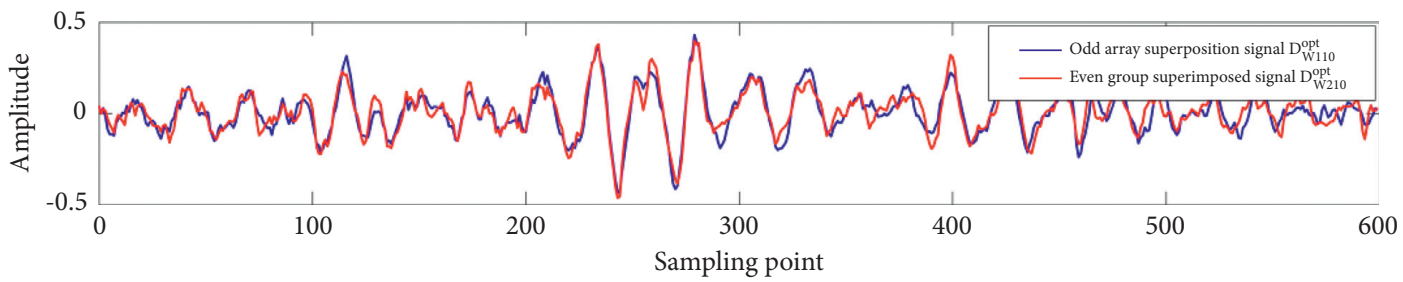

FIGURE 12: The superimposed signals of the odd group and even group after superimposing 10 times with the improved DSM. 

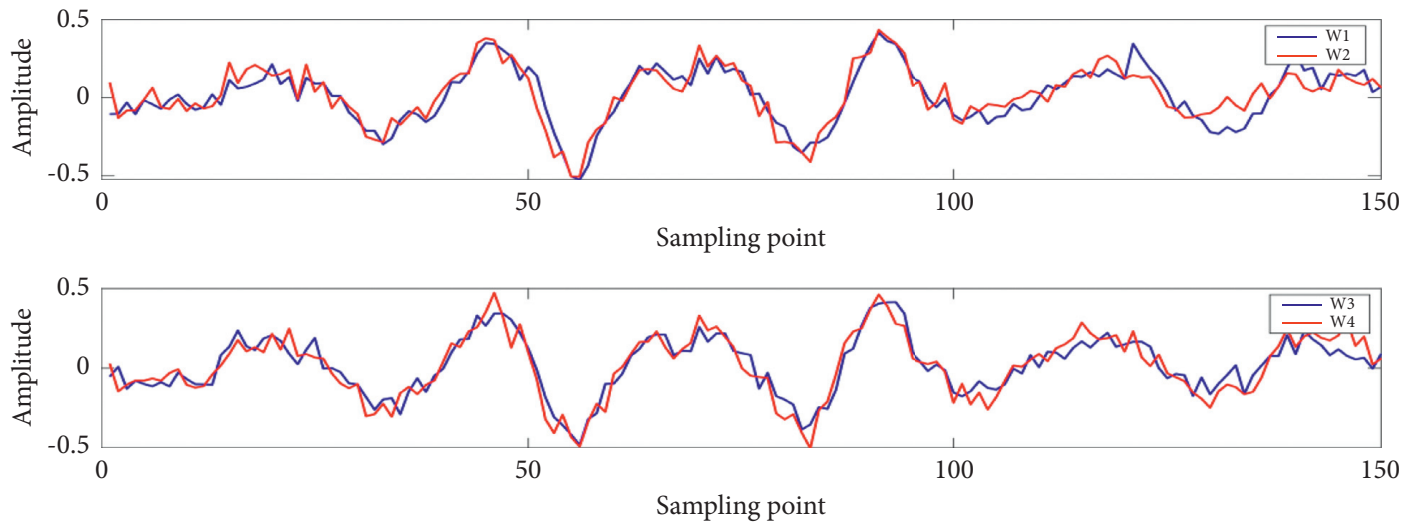

FIGURE 13: Fault components extracted accurately. $W_{1}, W_{2}, W_{3}$, and $W_{4}$ are fault components accurately extracted from $D_{W 114}^{\text {opt }}, D_{W 214}^{\text {opt }}$, $D_{W 110}^{\mathrm{opt}}$, and $D_{W 210}^{\mathrm{opt}}$, respectively.
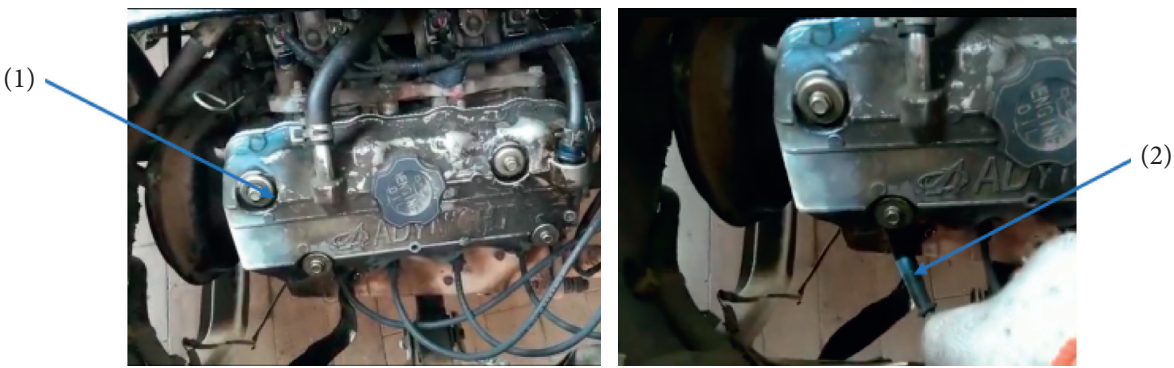

FIGURE 14: The physical map of the first cylinder lacking of the car engine. (1) Automotive engine; (2) the first cylinder line.

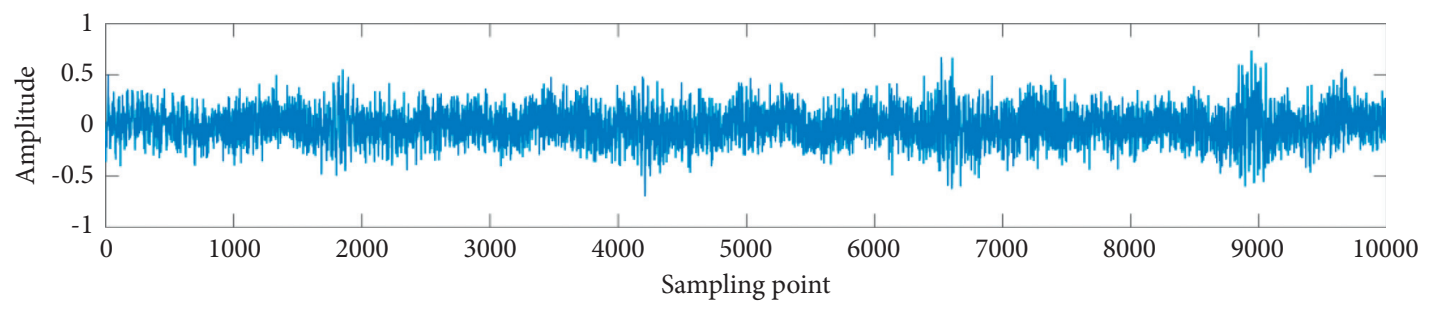

FIGURE 15: Engine abnormal noise signal of the first cylinder lacking.

TABLE 5: The sampling point corresponding to the fault feature points of each period.

\begin{tabular}{lcccccccccccc}
\hline$j$ & 1 & 2 & 3 & 4 & 5 & 6 & 7 & 8 & 9 & 10 & 11 & 12 \\
Sampling point $\left(n_{j j}\right)$ & 1794 & 4204 & 6567 & 8885 & 11161 & 13606 & 16002 & 18301 & 20798 & 23331 & 25749 & 28247 \\
\hline
\end{tabular}

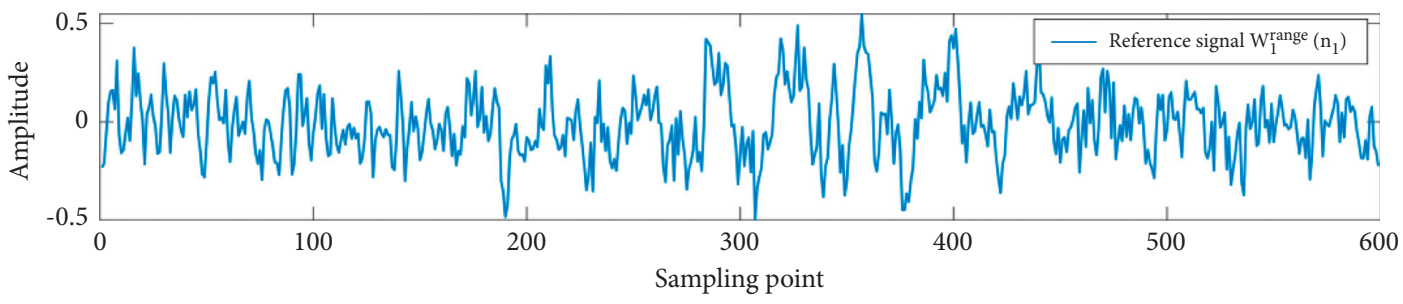

FIGURE 16: Reference signal $W_{1}^{\text {range }}\left(n_{1}\right)$.

TABLE 6: The optimal offset $\tau_{j}^{\mathrm{opt}}$ of $W_{j}^{\mathrm{fra}}\left(n_{j}+\tau_{j}\right)$.

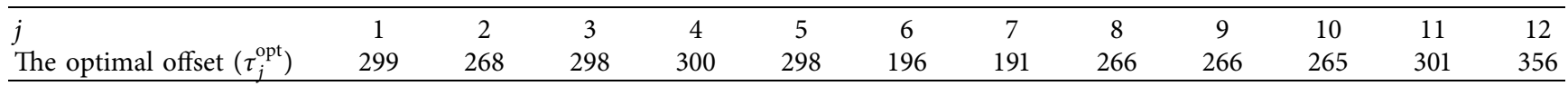




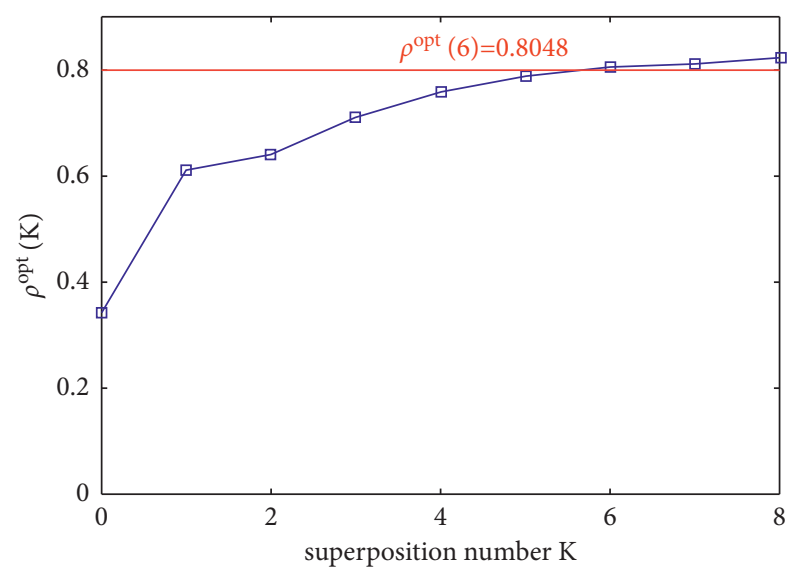

FIGURE 17: The variation curve of $\rho^{\text {opt }}(K)$ with the increase of the superimposition number $K$.

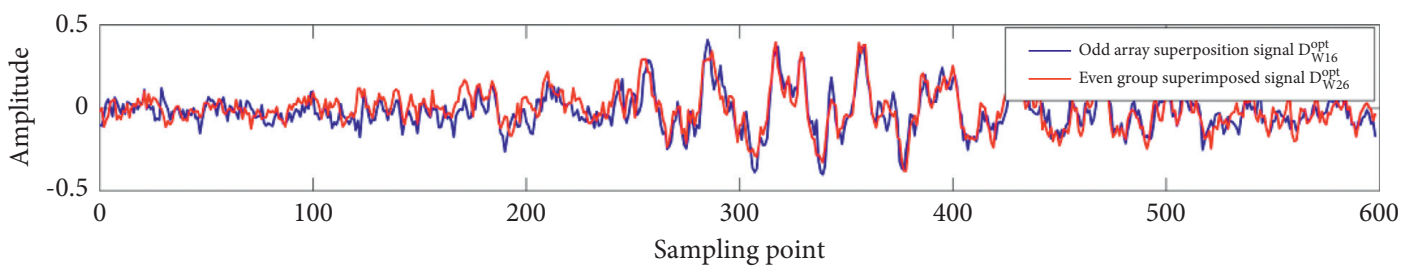

FIGURE 18: Odd group and even group superposition signals after 6-time superposition.

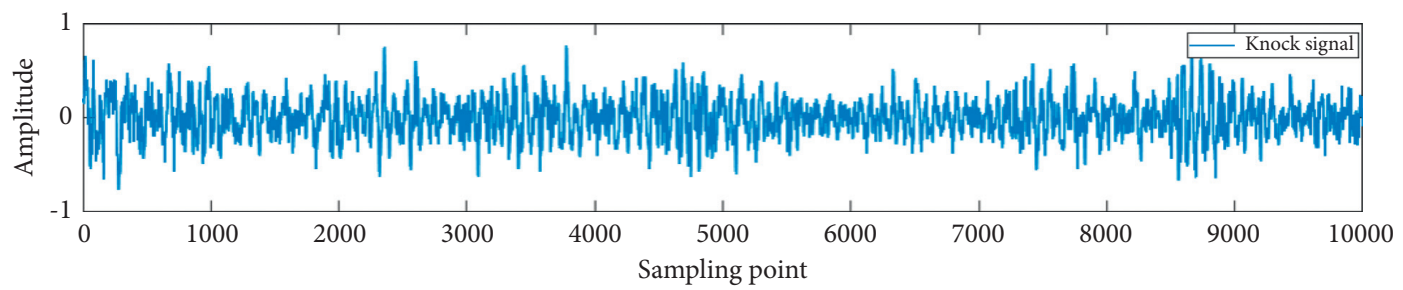

FIGURE 19: Engine knocking signal.

TABLE 7: The sampling point corresponding to the fault feature points of each period.

\begin{tabular}{|c|c|c|c|c|c|c|c|c|c|c|c|c|}
\hline$j$ & 1 & 2 & 3 & 4 & 5 & 6 & 7 & 8 & 9 & 10 & 11 & 12 \\
\hline Sampling point $\left(n_{j j}\right)$ & 8617 & 21164 & 25307 & 29327 & 34003 & 45681 & 49581 & 53832 & 57872 & 61933 & 70294 & 78110 \\
\hline
\end{tabular}

TABLE 8: The optimal offset $\tau_{j}^{\text {opt }}$ of $W_{j}^{\text {fra }}\left(n_{j}+\tau_{j}\right)$.

\begin{tabular}{|c|c|c|c|c|c|c|c|c|c|c|c|c|}
\hline$j$ & 1 & 2 & 3 & 4 & 5 & 6 & 7 & 8 & 9 & 10 & 11 & 12 \\
\hline The optimal offset $\left(\tau_{j}^{\mathrm{opt}}\right)$ & 299 & 228 & 17 & 389 & 167 & 244 & 442 & 236 & 234 & 234 & 447 & 232 \\
\hline
\end{tabular}

\subsection{Fault Component Extraction of Engine Knocking.} Figure 19 shows the engine knock signal due to the low gasoline label. The $\mathrm{db} 5$ wavelet is selected as the mother wavelet to perform CWT on the knocking signal. The timefrequency points of the two diagonally paired of the rectangular window $R$ are set as $(0,0),(0.0227,4000)$, respectively. The hard threshold of the wavelet coefficient $\lambda=2.8, \sigma=300$, $b=299$, and the subset $L$ of the offset $\tau_{j}$ is set as $\{0: 598\}$.
Table 7 shows the sampling points corresponding to the fault feature points. Due to the large value of hard threshold $\lambda$ of wavelet coefficient, the feature points of fault components do not show periodic feature, but the interval of sampling points between each two feature points is approximately 4000 times. Table 8 shows the optimal offset $\tau_{j}^{\text {opt }}$ of each $W_{j}^{\mathrm{fra}}\left(n_{j}+\tau_{j}\right)$, and $W_{j}^{\mathrm{fra}}\left(n_{j}+\tau_{j}^{\mathrm{opt}}\right)$ is obtained according to $\tau_{j}^{\text {opt }}$. Figure 20 shows the variation curve of the 


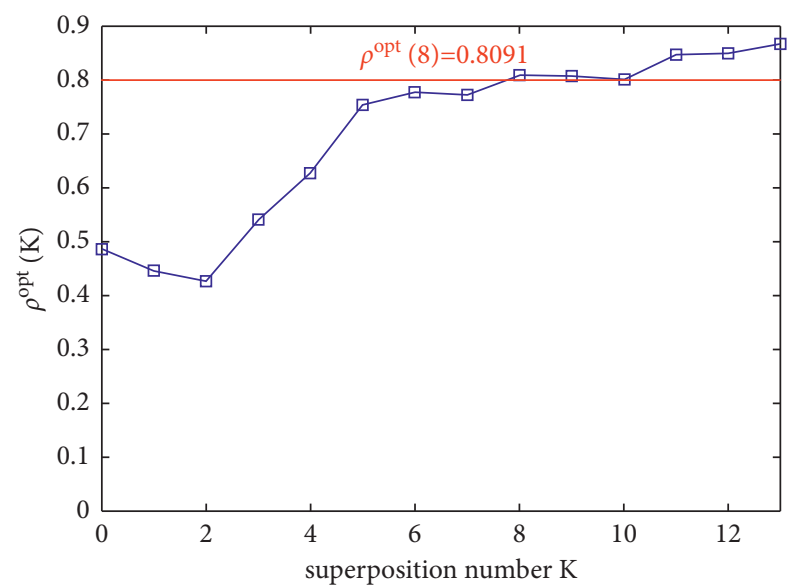

FIGURE 20: The variation curve of $\rho^{\text {opt }}(K)$ with the increase of the superimposition number $K$.

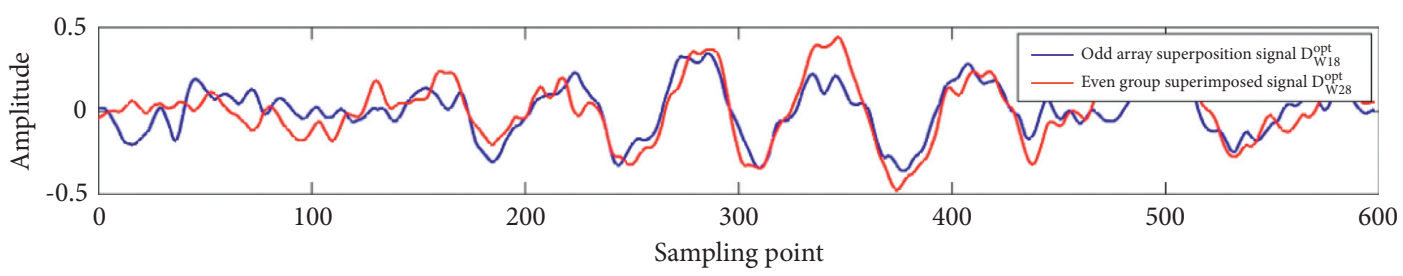

FIGURE 21: Odd group and even group superposition signals after 6-time superposition.

correlation coefficient $\rho^{\text {opt }}(K)$ of the superposition signals of the odd group and even group with the number of superpositions $K$. When $K=6, \rho^{\text {opt }}(8)=0.8091$, which is greater than the threshold $M$, and stopping superimposition, $D_{W 18}^{\mathrm{opt}}$ and $D_{W 28}^{\text {opt }}$ of the fault components after noise reduction is got, as shown in Figure 21.

\section{Experimental Results Analysis}

In order to further verify the effectiveness of this method, the experimental results of this method and the improved DSM are compared by using the knocking cylinder experiment of automobile engine, and then the effectiveness of this method is verified. In this method, odd group signals and even group of engine knocking cylinder signals are superimposed 14 times, and the correlation between the noise reduction signals $D_{W 114}^{\mathrm{opt}}$ and $D_{W 214}^{\mathrm{opt}}$ is 0.8030 . Then, the noise reduction processing and extraction of engine knocking cylinder failure component are carried out by using the improved DSM. When odd group signals and even group signals are superposed 10 times, the correlation between the noise reduction signals $D_{W 110}^{\mathrm{opt}}$ and $D_{W 210}^{\mathrm{opt}}$ is 0.8020 . Finally, the fault components $W_{1}, W_{2}, W_{3}$, and $W_{4}$ are accurately extracted from noise reduction signals of $D_{W 114}^{\text {opt }}, D_{W 214}^{\text {opt }}$, $D_{W 110}^{\text {opt }}$, and $D_{W 210}^{\text {opt }}$, where the correlation between $W_{1}$ and $W_{3}$ is 0.9029 , and the correlation between $W_{2}$ and $W_{4}$ is 0.9290 . The results show that this method can further replace the use of encoders and achieve the same noise reduction effect as the improved DSM.

In the engine cylinder lacking experiment, this method is used to reduce the noise of fault components. The correlation between the noise reduction signal $D_{W 16}^{\text {opt }}$ and $D_{W 26}^{\text {opt }}$ obtained by superposition of odd group signals and even group signals for 6 times is 0.8048 . In the engine knock experiment, due to the relatively small setting of hard threshold $\lambda$, some fault feature points are not selected accurately, resulting in the correlation of superposition signals of odd and even groups after superposition for 2 and 3 times gradually decreasing, as shown in Figure 20 . However, with the gradual increase in the number of superpositions, the correlation between $D_{W 18}^{\mathrm{opt}}$ and $D_{W 28}^{\mathrm{opt}}$ obtained by superposition of odd group and even group for 8 times is 0.8091 .

From the analysis results, the method can replace the improved DSM, effectively reduce the noise, and extract the fault component of the engine abnormal noise signal. By increasing the threshold $M$ and superimposition number $K$, the accuracy of the extracted fault components can be improved. The method can also use the extracted fault components to establish a database of automobile engine faults, which lays a foundation for the later diagnosis and classification of automobile engine faults.

\section{Conclusion}

Althoughthe improved DSM can automatically find the starting superposition point andsuperposition length of the periodic signal, it needs to use the encoder toassist in finding the starting superposition point. In practical applications, many occasions are not suitable for the use of encoders, which limits the applicability of DSM. Therefore, based on the improved DSM, this paper proposes an adaptive noise reduction method of automobile engine abnormal noise 
signal, which cancels the use of the encoder. Using this method, the fault components of knocking cylinder, cylinder lacking, and knocking are extracted from the abnormal sound signal of automobile engine, and the effectiveness of the method is verified. The method can adaptively obtain the starting superposition point and superposition length from the engine abnormal sound signal and determine the number of superpositions, which replaces the encoder in the improved DSM to determine the starting position of the automobile engine impact fault and greatly improves the applicability of DSM. However, when the hard threshold $\lambda$ is set to a small value, the selection of fault feature points will be inaccurate, and then the selected fault components will be biased, resulting in inaccurate noise reduction signals. The specific problem of hard threshold setting will be further studied. When the starting superposition point is selected, the accuracy is reduced compared with the improved DSM, resulting in more superposition times than the improved DSM. However, according to the superposition results, the increase of superposition times is within an acceptable range.

In the future, the hard threshold $\lambda$ setting problem will be further improved, and the corresponding fault component database will be established. The fault type will be determined by comparing the extracted fault component with the fault component in the database.

\section{Data Availability}

The data used to support the findings of this study are available from the corresponding author upon request.

\section{Conflicts of Interest}

The authors declare that there are no conflicts of interest regarding the publication of this study.

\section{Acknowledgments}

This work was funded in part by the National Key Research and Development Program under Grants 2018YFC0810406 and 2018YFC0810404, in part by the Fundamental Research Funds for the Central Universities under Grant 3132019118, and in part by the National Natural Science Foundation of China under Grant U1908228.

\section{References}

[1] K. Jafarian, M. Darjani, and Z. Honarkar, "Vibration analysis for fault detection of automobile engine using PCA technique," in Proceedings of the 2016 4th International Conference on Control, Instrumentation, and Automation (ICCIA), pp. 372-376, Qazvin, Iran, January 2016.

[2] J. Mohammadpour, M. Franchek, and K. Grigoriadis, "A survey on diagnostic methods for automotive engines," International Journal of Engine Research, vol. 13, no. 1, pp. 4164, 2012.

[3] X. L. Liang, T. Chen, and X. D. Yang, "Application of fuzzy fault tree analysis in the fault diagnosis for automobile engine system," in Proceedings of the 2017 9th International
Conference on Modelling, Identification and Control (ICMIC), pp. 711-716, Kunming, China, July 2017.

[4] L. Zhang, Y. Shi, and L. Ren, "Humanoid extraction of abnormal engine sounds by using ICA-R and VANC," in Proceedings of the 2012 International Conference on Systems and Informatics (ICSAI2012), pp. 1687-1692, Yantai, China, May 2012.

[5] J. Xie, J. G. Colonna, and J. L. Zhang, "Bioacoustic signal denoising: a review," Artificial Intelligence Review, vol. 54, no. 3, pp. 1-23, 2020.

[6] A. Azarang and N. Kehtarnavaz, "A review of multi-objective deep learning speech denoising methods," Speech Communication, vol. 122, pp. 1-10, 2020.

[7] H. Wang and Z. Ma, "Research of acoustic signal de-noising using wavelet transform," in Proceedings of the 2009 2nd International Congress on Image and Signal Processing, pp. 1-3, Tianjin, China, October 2009.

[8] S. V. P. S. Nidadavolu, S. K. Yadav, and P. K. Kalra, "Condition monitoring of internal combustion engines using empirical mode decomposition and morlet wavelet," in Proceedings of the 2009 Proceedings of 6th International Symposium on Image and Signal Processing and Analysis, pp. 65-72, Salzburg, Austria, September 2009.

[9] X. Chen and Y. Yang, "De-noising for vibration signals based on local mean decomposition," in Proceedings of the IECON 2017 - 43rd Annual Conference of the IEEE Industrial Electronics Society, pp. 3298-3303, Beijing, November 2017.

[10] N. Dayong, J. Yuhua, S. Hongyu et al., "Separation method of impulsive fault component for gasoline engine based on acoustic signal analysis," Shock and Vibration, vol. 2019, Article ID 8573479, 15 pages, 2019.

[11] H. Qiu, J. Lee, J. Lin, and G. Yu, "Wavelet filter-based weak signature detection method and its application on rolling element bearing prognostics," Journal of Sound and Vibration, vol. 289, no. 4-5, pp. 1066-1090, 2006.

[12] M. Li, "Weak fault signal detection of rolling bearing," in Proceedings of the 2011 International Conference on Transportation, Mechanical, and Electrical Engineering (TMEE), pp. 540-543, Changchun, China, December 2011.

[13] A. Moshrefi, H. Aghababa, and O. Shoaei, "Knock signal denoising employing a new time domain method," in Proceedings of the 2017 15th IEEE International New Circuits and Systems Conference (NEWCAS), pp. 37-40, Strasbourg, France, June 2017.

[14] W. Kai, D. Shaobo, Y. Zilin, and S. Shijie, "Application of wavelet threshold denoising on bearing fault diagnosis," in Proceedings of the 2019 Chinese Control and Decision Conference (CCDC), pp. 1980-1985, Nanchang, China, June 2019.

[15] X. Wen and L. You, "A novel rolling bearing fault detection method based on wavelet transform and empirical mode decomposition," in Proceedings of the 2019 Chinese Control Conference (CCC), pp. 5024-5027, Guangzhou, China, July 2019.

[16] N. E. Huang, Z. Shen, S. R. Long et al., "The empirical mode decomposition and the Hilbert spectrum for nonlinear and non-stationary time series analysis," Proceedings of the Royal Society of London. Series A: Mathematical, Physical and Engineering Sciences, vol. 454, no. 1971, pp. 903-995, 1998.

[17] B. Sun, S. Zhou, and C. Wang, "Application of wavelet soft threshold denoising algorithm based on EMD decomposition in vibration signals," in Proceedings of the 2019 6th International Conference on Systems and Informatics (ICSAI), pp. 7-11, Shanghai, China, November 2019. 
[18] G. Ren and Z. Liu, "An improved EMD adaptive denoising and feature extraction algorithm," in Proceedings of the 2019 IEEE International Conference on Signal Processing, Communications and Computing (ICSPCC), pp. 1-4, Dalian, China, September 2019.

[19] Z. Wu and N. E. Huang, "Ensemble empirical mode decomposition: a noise-assi-sted data analysis method," $A d$ vances in Adaptive Data Analysis, vol. 1, no. 1, pp. 1-41, 2009.

[20] X. Gao, H. Wen, and Pu Wang, "A new method of bearing fault diagnosis based on LMD and wavelet denoising," in Proceedings of the 2017 29th Chinese Control and Decision Conference (CCDC), pp. 4155-4162, Chongqing, 2017.

[21] N. Dayong, S. Changle, G. Yongjun, Z. Zengmeng, and H. Jiaoyi, "Extraction of fault component from abnormal sound in diesel engines using acoustic signals," Mechanical Systems and Signal Processing, vol. 75, pp. 544-555, 2016.

[22] N. Dayong, S. Hongyu, X. Aoyu, G. Yongjun, D. Hongwei, and H. Jiaoyi, "Adaptive noise reduction method of synchronous hydraulic motor acoustic signal based on improved dislocation superposition method," IEEE Access, vol. 8, pp. 37161-37172, 2020.

[23] J. Yuhua, Research and Experiment on Extraction of Engine Fault Components Based on Abnormal Sound Analysis, Dalian Maritime University, Dalian, China, 2019. 